\title{
用于多天线通信系统的自适应波束形成与 空时块码相结合方案
}

林敏 $\mathbb{1 0}^{*}$, 李敏 ${ }^{\circledR}$, 杨绿溪 ${ }^{\circledR}$, 李斌

(1) 东南大学信息科学与工程学院, 南京 210096 ;

(2) 南京电讯技术研究所，南京 210007 ;

(3) 华为技术有限公司, 深圳 518129

*E-mail: linmin163@163.com

收稿日期: 2007-08-11; 接受日期: 2007-11-11

国家重点基础研究发展计划(批准号: 2007CB310603)、国家自然科学基金(批准号: 60672093, 60496310)、国家高技术 研究发展计划(批准号: 2007AA01Z262)、江苏省自然科学基金(批准号: BK2005061)和华为高校基金资助项目

摘要针对多天线通信系统, 提出了一种将自适应波束形成( $\mathrm{ABF})$ 与空 时块码(STBC)相结合的下行链路发射方案。首先基于 STBC 所产生的等效 加权加性 Gauss 白噪声(AWGN)信道模型, 求得接收平均信噪比(SNR)最 大化条件下的发射端自适应波束形成权矢量. 接着以常用的 3 大类调制方 式下的误符号率(SER)上界为准则，设计出各个波束间的最优功率分配算

关键词

自适应波束形成

空时块码

发射方案

多天线通信 法。该发射方案实际上是对应于信道自相关矩阵的特征波束形成，同时结 合空时编码以获得分集增益，从而提高无线通信下行链路的性能. 此外, 基于矩生成函数(MGF)和 Gauss-Chebyshev 积分, 还提出了一种简单而精 确的数值计算方法，用来分析采用新方案的通信系统在常用调制方式下的 性能. 最后计算机仿真结果验证了新方案的性能优于常用的空时块码和现 有相关文献上介绍的方法.

众所周知, 在无线通信系统中发射端和接收端同时采用多根天线能有效地克服无线信道 的局限性, 从而提高通信的容量和可靠性 ${ }^{[1 \sim 3]}$. 然而, 在一个实际的系统中, 尺寸和实现的复 杂度往往决定了作为接收部分的小型手持移动终端只能安装 1 2 根天线. 因此在基站采用阵 列天线被认为是更加现实和有效的方法.

在移动通信领域, 基于天线阵的波束形成技术已经得到了广泛的研究(参见文献 $[4,5]$ 及其 参考文献). 波束形成的基本原理是基站上不同天线单元发射的信号在接收端同相叠加，从而 
获得比单根天线好得多的性能. 此外, 波束形成还具有空间匹配滤波或干扰抑制的能力. 然而, 波束形成要求发射端必须准确已知信道状态信息(CSI). 与波束形成正好相反, 发射分集技术 不需要任何的信道参数信息. 尽管文献[6]提出的空时格码能获得良好的性能, 但其译码复杂 度随着分集阶数和传输速率呈指数增长. 而首先由文献[7]针对两根发射天线提出的, 随后经 文献[8]推广到多根天线的正交空时块码, 由于只需在接收端作线性处理就能获得最大似然检 测性能，因此成为了许多无线通信系统的标准.

尽管发射端准确已知 CSI 的假设在许多实际的系统中不能实现, 但是文献[9]以互信息量 为出发点, 文献 $[10,11]$ 以信道容量为出发点, 均证明了利用部分信道信息能进一步提高多天 线通信系统的性能, 从而极大地推动了 BF 和 STBC 相结合方案的研究进展. 其中文献[12,13] 基于信道均值和方差反馈, 以成对差错概率(PEP)为准则, 针对特定的 STBC 设计出了最优的 预编码方案. 以文献[12]为基础, 文献[14]进一步提出了将一维波束形成和 Alamouti 空时块码 相结合的方案, 以提高发射天线数大于 2 的情形下系统的误码性能. 然而由于只能形成一个波 束, 这 2 种方案均不能获得分集增益. 跟它们不同的是, 文献 $[15,16]$ 提出的空时发射方案由于 在形成 2 个特征波束的基础上再进行 Alamouti 空时编码, 因此能同时获得分集增益和阵列增 益. 然而这类方法的缺点是不能根据信道状况进行自适应功率分配, 从而导致其性能在许多 情形下并非最优. 另外这几篇文献均没有对这种空时发射方案的性能进行足够的理论分析. 除此之外, 基于接收端逐个符号检测, 文献[17]针对 3 大类最为常用的调制方式设计出了最优 发射分集预编码方案. 而文献中所谓的与 STBC 相结合实际上是要起到在不增加系统额外开 销的条件下提高数据传输速率的目的. 需要指出的是该文献在系统建模时没有将空时块码的 影响考虑进去, 并且所提出的方案仅适用于对频谱利用率没有严格要求的场合, 这一点很显 然不符合未来移动通信的发展方向.

在这种情形下，我们提出了一种适用于多天线通信系统的空时发射方案. 该方案首先基 于 STBC 所产生的等效加权 AWGN 信道模型, 求得接收平均信噪比最大时的发射端波束形成 权矢量. 接着以 3 大类调制方式下的误符号率上界为准则, 得到了功率在各个波束间进行自适 应分配的算法, 从而完成 ABF 与 STBC 相结合的发射方案的设计. 为了验证新方案的优越性, 我们还提出了一种数值分析方法. 由于它采用了 MGF 和 Gauss-Chebyshev 积分, 因此能高效 且精确地计算出 3 大类常用调制方式下的系统误符号率. 这 3 类调制方式是: $M$ 元脉冲幅度调 制( $M-\mathrm{PAM}) 、 M$ 元相移键控( $M-\mathrm{PSK})$ 和 $M$ 元正方形正交幅度调制( $M-\mathrm{QAM})$.

本文的其余部分组织如下: 第 1 节首先介绍了系统模型; 第 2 节详细地描述了 $\mathrm{ABF}$ 与 STBC 相结合的空时发射方案; 第 3 节针对 3 大类常用调制方式, 推导了基于 MGF 和 Gauss-Chebyshev 积分的性能分析方法; 最后在第 4 和第 5 节分别给出了仿真结果和结论.

符号说明:黑体表示矩阵或矢量, $|\cdot|$ 表示绝对值, $\|\cdot\|_{F}$ 表示矩阵的 Frobenius 范数, $E[\cdot]$ 表 示取统计平均值, $\operatorname{diag}(\cdot)$ 表示对角矩阵, $(\cdot)^{*},(\cdot)^{\mathrm{T}}$, 和 $(\cdot)^{\mathrm{H}}$ 分别表示共轭, 转置和 Hermitian 转 置. $\chi_{n}^{2}$ 代表自由度为 $n$ 的卡方分布, $\ln (\cdot)$ 代表自然对数函数, $\exp (\cdot)$ 代表指数函数, $\tan (\cdot)$ 代表 正切函数. $\delta(i-j)$ 表示 Kronecker delta 函数, 它在 $i=j$ 时为 1 , 在 $i \neq j$ 为 $0, C^{M \times N}$ 表示 $M \times N$ 
的复矩阵, $\aleph_{C}\left(m, \sigma^{2}\right)$ 表示均值为 $m$ 方差为 $\sigma^{2}$ 的复 Gauss 分布.

\section{1 系统模型}

跟文献[15 17]一样, 为了将问题阐述清楚, 我们只讨论由 $N$ 根发射天线和 1 根接收天线 组成的无线通信系统. 当然本文提出的方案和性能分析方法很容易推广到多根接收天线的情 形. 在一个实际的系统中, 利用信道估计技术接收端可以准确获得当前的 CSI, 而发射端可以 通过反馈 ${ }^{[17,18]}$ 或矩阵变换 ${ }^{[19]}$ 的方法得到信道的自相关阵.

\section{1 信道模型}

针对多天线通信系统, 目前已经提出了多种信道模型. 总的来看它们可分成 3 大类: 射线 跟踪模型、散射模型和相干模型. 本文选择基于散射簇的平衰落信道模型, 并且假设时延扩展 相对于符号周期可以忽略不计. 在这种信道模型下, 我们假设散射簇中的 $L$ 条多径信号的离 开角 $(\mathrm{AOD})$ 在以 $\theta_{c}$ 为均值、以 $\Delta \theta$ 为角度扩展的范围内均匀分布, 那么瞬时的信道响应矢量可 以表示为

$$
\boldsymbol{h}(t)=\sum_{l=1}^{L} \rho_{l}(t) \boldsymbol{a}\left(\theta_{l}\right),
$$

其中 $\theta_{l}$ 和 $\rho_{l}(t)$ 分别表示第 $l$ 径信号的 $\mathrm{AOD}$ 和衰落系数. 不失一般性, $\rho_{l}(t)$ 通常被建模为服从 $\aleph_{C}(0,1)$ 分布的随机变量. 如果发射端选择均匀线阵(ULA), 那么阵列导引矢量为

$$
\boldsymbol{a}\left(\theta_{l}\right)=\left[1, \exp \left(\mathrm{j} \beta d \cos \theta_{l}\right), \exp \left(\mathrm{j} 2 \beta d \cos \theta_{l}\right), \ldots, \exp \left(\mathrm{j}(N-1) \beta d \cos \theta_{l}\right)\right]^{\mathrm{T}} .
$$

上式中的 $d$ 为相邻阵元间距离, $\beta$ 为波数, 即 $\beta=2 \pi / \lambda, \lambda$ 表示载波波长. 更进一步, 信道的自 相关阵可由下面的式子得到:

$$
\boldsymbol{R}=E\left[\boldsymbol{h}(t) \cdot \boldsymbol{h}^{\mathrm{H}}(t)\right]=\sum_{l=1}^{L} E\left[\left|\rho_{l}(t)\right|^{2}\right] \cdot \boldsymbol{a}\left(\theta_{l}\right) \cdot \boldsymbol{a}^{\mathrm{H}}\left(\theta_{l}\right) .
$$

为了使公式更加简洁, 在后面的小节中我们省略了自变量 $t$.

\section{2 发射 BF 结合 STBC}

图 1 表示一个 BF 与 STBC 相结合的无线通信系统框图. 其工作原理如下: 首先将待发射 的调制信号 $s$ 进行空时编码, 得到 $P(P<N)$ 路并行数据. 根据文献[8], 空时编码后的发射矩阵 通常可以表示为

$$
\boldsymbol{S}=\left[\begin{array}{cccc}
s_{11} & s_{12} & \cdots & s_{1 T} \\
s_{21} & s_{22} & \cdots & s_{2 T} \\
\vdots & \vdots & & \vdots \\
s_{P 1} & s_{P 2} & \cdots & s_{P T}
\end{array}\right],
$$

其中 $T$ 表示对 $K$ 个符号进行编码需要的时隙数, $s_{i j}(i \leqslant P, j \leqslant T)$ 表示调制信号及其共轭的线 性组合. 接着每一路并行数据流都经过发射波束形成, 其输出信号相加后由 $N$ 个阵元同时发 
射出去. 在某一时刻 $n T$, 与第 $n$ 个输入数据块相对应的发射信号矩阵 $\boldsymbol{X}_{n T}(N \times T)$ 可以表示成

$$
\boldsymbol{X}_{n T}=\boldsymbol{W S} \text {. }
$$

式中 $\boldsymbol{W}=\left[\boldsymbol{w}_{1}, \boldsymbol{w}_{2}, \ldots, \boldsymbol{w}_{P}\right], \boldsymbol{w}_{i}=\left[w_{i 1}, w_{i 2}, \ldots, w_{i N}\right]^{\mathrm{T}}$ 表示第 $i$ 个波束形成器的权矢量. 在保证发射 总功率不变的条件下, 它们满足下面的关系式

$$
\begin{aligned}
& \boldsymbol{w}_{i}^{\mathrm{H}} \boldsymbol{w}_{i}=f_{i}^{2} \quad(i=1,2, \ldots, P), \\
& \sum_{i=1}^{P} f_{i}^{2}=1,
\end{aligned}
$$

其中 $f_{i}^{2}(i=1,2, \ldots, P)$ 表示波束间功率分配系数. 可见设计 BF 与 STBC 相结合的发射方案的关 键在于确定波束形成权矢量 $\boldsymbol{w}_{i}$ 和相应的功率分配系数 $f_{i}^{2}$.

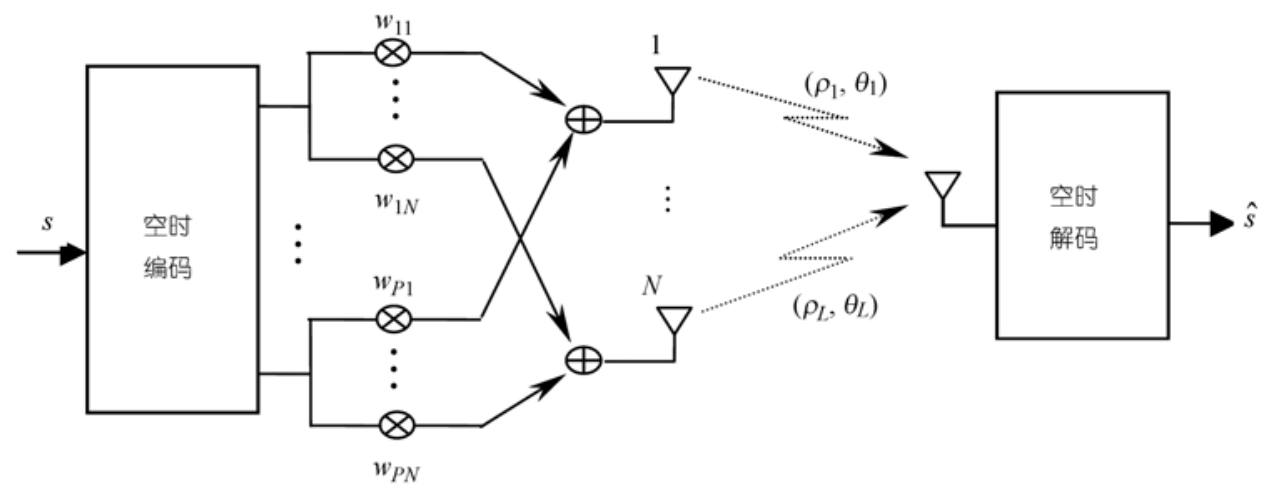

图 1 BF 与 STBC 相结合的无线通信系统框图

\section{2 新的空时发射方案}

在本节, 以接收端达到最大的平均 SNR 和最小的 SER 上界为设计准则, 我们提出了 $\mathrm{ABF}$ 与 STBC 相结合的空时发射方案.

\section{1 最佳波束形成权矢量}

根据(5)式，由天线阵发射出去的信号经过衰落信道后，在某一时刻 $n T$ 的接收信号矢量 $\boldsymbol{Y}_{n T}(1 \times T)$ 可以表示成

$$
\boldsymbol{Y}_{n T}=\boldsymbol{h}^{\mathrm{H}} \boldsymbol{W} \boldsymbol{S}+\boldsymbol{V}_{n T},
$$

其中 $\boldsymbol{V}_{n T}(1 \times T)$ 表示接收噪声矢量, 它的每个元素为独立同分布(i.i.d.)的, 且服从 $\aleph_{C}\left(0, N_{0}\right)$ 分 布的随机变量. 令

$$
g_{i}=\boldsymbol{h}^{\mathrm{H}} \boldsymbol{w}_{i}(i=1,2, \ldots, P) .
$$

我们不难发现通过选择 $\boldsymbol{w}_{i}$ 可以产生 $P$ 个等效的衰落信道. 定义新的信道矩阵为

$$
\boldsymbol{G}=\left[g_{1}, g_{2}, \ldots, g_{P}\right]=\boldsymbol{h}^{\mathrm{H}} \boldsymbol{W} .
$$

那么基于 STBC 所产生的等效加权 AWGN 信道模型 [20], 解码后的信号矢量可以表示成 


$$
\boldsymbol{y}_{n T}=\frac{1}{R}\|\boldsymbol{G}\|_{F}^{2} \boldsymbol{s}_{n T}+\boldsymbol{v}_{n T},
$$

其中 $\boldsymbol{y}_{n T}$ 表示对接收信号矢量 $\boldsymbol{Y}_{n T}$ 进行空时解码后得到的 $K \times 1$ 复矢量, $\boldsymbol{s}_{n T}(K \times 1)$ 表示输入信 号矢量, 而 $\boldsymbol{v}_{n T}$ 表示每个元素 i.i.d. 且均值为 0 方差为 $\|\boldsymbol{G}\|_{F}^{2} N_{0} / R$ 的 $K \times 1$ 复 Gauss 噪声矢量. $R$ 表示 STBC 的码率, 即 $R=K / T$. 于是接收端的瞬时 SNR 为

$$
\gamma=\frac{R E_{S}}{R N_{0}}\|\boldsymbol{G}\|_{F}^{2} \triangleq \gamma_{s} \cdot\|\boldsymbol{G}\|_{F}^{2},
$$

其中 $\gamma_{S}=E_{S} / N_{0}$ 代表发射符号信噪比. 根据 STBC 的基本原理, 只有在信道独立不相关的条件 下才有最好的性能, 于是我们得到 $E\left[g_{i} \cdot g_{j}^{*}\right]=0, \forall(i \neq j)$. 同时, 在发射端已知信道自相关阵 的条件下, 应该以接收端输出平均信噪比最大化为目标函数. 于是不难得到下面约束条件下 的最优化问题

$$
\begin{aligned}
& J=\max _{\boldsymbol{w}_{1}, \boldsymbol{w}_{2}, \ldots, \boldsymbol{w}_{P}} E\left[\|\boldsymbol{G}\|_{F}^{2}\right], \\
& \text { s.t. } E\left[g_{i} \cdot g_{j}^{*}\right]=0, \quad \forall(i \neq j), \\
& \boldsymbol{w}_{i}^{\mathrm{H}} \boldsymbol{w}_{i}=f_{i}^{2}, \quad \sum_{i=1}^{P} f_{i}^{2}=1 .
\end{aligned}
$$

我们在附录 $\mathrm{A}$ 中证明了上述最优化问题的解由下面的式子给出

$$
\begin{aligned}
& J=\sum_{i=1}^{P} \lambda_{i} f_{i}^{2}, \\
& \boldsymbol{w}_{i}^{\mathrm{opt}}=f_{i} \boldsymbol{u}_{i} \quad(i=1,2, \ldots, P) .
\end{aligned}
$$

其中 $\lambda_{i}$ 和 $\boldsymbol{u}_{i}$ 是矩阵 $\boldsymbol{R}$ 的特征值和对应的特征矢量, 即

$$
\boldsymbol{R}=E\left[\boldsymbol{h} \boldsymbol{h}^{\mathrm{H}}\right]=\boldsymbol{U} \Sigma \boldsymbol{U}^{\mathrm{H}}=\left[\boldsymbol{u}_{1}, \boldsymbol{u}_{2}, \ldots, \boldsymbol{u}_{N}\right] \operatorname{diag}\left(\lambda_{1}, \lambda_{2}, \ldots, \lambda_{N}\right)\left[\boldsymbol{u}_{1}, \boldsymbol{u}_{2}, \ldots, \boldsymbol{u}_{N}\right]^{\mathrm{H}} .
$$

不失一般性, 上式中的 $N$ 个特征值按非递增的顺序排列, 即 $\lambda_{1} \geqslant \lambda_{2} \geqslant \cdots \geqslant \lambda_{N}$. 至此, 我们已 经证明了在接收端获得最大平均 SNR 的条件下, 发射端波束形成权矢量恰好等于信道自相关 阵的前 $P$ 个特征矢量. 跟文献[11]提出的以信道容量为准则以及文献[12]提出的以 PEP 为准则 的方法相比，本文方法在物理意义非常明确的同时，数学推导简单易懂.

\section{2 自适应功率分配}

在 2.1 小节, 我们计算发射端最佳波束形成权矢量时, 假设 $P$ 和 $f_{i}^{2}$ 的值是已知的. 而在本 小节, 我们将以 3 大类常用调制方式下的 SER 上界为准则讨论自适应功率分配算法, 即计算波 束个数 $P$ 和功率分配系数 $f_{i}^{2}(i=1,2, \ldots, P)$ 的值. 根据文献[21], 基于 STBC 所产生的等效加权 AWGN 信道模型, 3 大类最为常用的调制方式, 即 $M$-PSK $(M \geqslant 4) 、 M-P A M$ 和 $M$-QAM $(M \geqslant 4)$ 的 SER 分别满足下面的式子 


$$
\begin{aligned}
& P_{S, \mathrm{PSK}} \approx 2 E\left[Q\left(\sqrt{2 \sin ^{2}\left(\frac{\pi}{M}\right) \gamma}\right)\right], \\
& P_{S, \mathrm{PAM}}=\frac{2(M-1)}{M} E\left[Q\left(\sqrt{\frac{6}{M^{2}-1} \gamma}\right)\right], \\
& P_{S, \mathrm{QAM}} \leqslant 4 E\left[Q\left(\sqrt{\frac{3}{M-1} \gamma}\right)\right],
\end{aligned}
$$

其中 $Q(\cdot)$ 表示 $Q$ 函数, 即 Gauss 概率密度函数尾部曲线下的面积函数. 利用 $Q$ 函数的一个上界 公式[22]

$$
Q(x) \leqslant \frac{1}{2} \exp \left(-\frac{x^{2}}{2}\right),
$$

同时考虑(12)和(13)式，我们可以用一个统一的式子将(17)式中的 SER 上界表示出来

$$
P_{S, \text { bound }}=a E\left[\exp \left(-b \gamma_{s}\|\boldsymbol{G}\|_{F}^{2}\right)\right]=a E\left[\exp \left(-b \gamma_{s} \sum_{i=1}^{P}\left|g_{i}\right|^{2}\right)\right],
$$

其中

$$
a=\left\{\begin{array}{ll}
1, & \text { for PSK, } \\
\frac{M-1}{M}, & \text { for PAM }, \\
2, & \text { for QAM, }
\end{array} \quad b= \begin{cases}\sin ^{2}\left(\frac{\pi}{M}\right), & \text { for PSK, } \\
\frac{3}{M^{2}-1}, & \text { for PAM }, \\
\frac{3}{2(M-1)}, & \text { for QAM. }\end{cases}\right.
$$

由于 $g_{i}$ 是独立同分布的复 Gauss 随机变量, 并且其均值为 0 , 方差为

$$
E\left[g_{i} \cdot g_{i}^{*}\right]=f_{i}^{2} \lambda_{i}(i=1,2, \ldots, P) .
$$

我们首先预白化 $g_{i}$, 即令

$$
g_{i}=f_{i} \sqrt{\lambda_{i}} \tilde{g}_{i}
$$

使得 $\tilde{g}_{i} \sim \aleph_{C}(0,1) \quad(i=1,2, \ldots, P)$. 设

$$
z_{i}=\left|\tilde{g}_{i}\right|^{2},
$$

那么 $z_{i}$ 是一个服从方差为 0.5 的 $\chi_{2}^{2}$ 分布, 其概率密度函数为

$$
p\left(z_{i}\right)=\exp \left(-z_{i}\right) .
$$

将(21) (23)式代入(19)式，可以得到

$$
P_{S, \text { bound }}=a E\left[\exp \left(-b \gamma_{s} \sum_{i=1}^{P} \lambda_{i} f_{i}^{2} z_{i}\right)\right]
$$




$$
\begin{aligned}
& =a \int_{0}^{\infty} \cdots \int_{0}^{\infty} \exp \left(-b \gamma_{s} \sum_{i=1}^{P} \lambda_{i} f_{i}^{2} z_{i}\right) \exp \left(-z_{1}\right) \cdots \exp \left(-z_{P}\right) \mathrm{d} z_{1} \cdots \mathrm{d} z_{P} \\
& =a\left\{\int_{0}^{\infty} \exp \left[-\left(b \gamma_{s} \lambda_{1} f_{1}^{2}+1\right) z_{1}\right] \mathrm{d} z_{1}\right\} \cdots\left\{\int_{0}^{\infty} \exp \left[-\left(b \gamma_{s} \lambda_{P} f_{P}^{2}+1\right) z_{P}\right] \mathrm{d} z_{P}\right\} .
\end{aligned}
$$

对上式做变量替换 $\left(b \gamma_{s} \lambda_{i} f_{i}^{2}+1\right) z_{i}=u$, 其第 $i$ 项

$$
\int_{0}^{\infty} \exp \left[-\left(b \gamma_{s} \lambda_{i} f_{i}^{2}+1\right) z_{i}\right] \mathrm{d} z_{i}=\int_{0}^{\infty} \frac{\exp (-u)}{\left(1+b \gamma_{s} \lambda_{i} f_{i}^{2}\right)} \mathrm{d} u=\frac{1}{1+b \gamma_{s} \lambda_{i} f_{i}^{2}},
$$

从而，整个(24)式可以简化为

$$
P_{S, \text { bound }}=a \frac{1}{1+b \gamma_{s} \lambda_{1} f_{1}^{2}} \cdots \frac{1}{1+b \gamma_{s} \lambda_{i} f_{i}^{2}} \cdots \frac{1}{1+b \gamma_{s} \lambda_{P} f_{P}^{2}}=a \prod_{i=1}^{P} \frac{1}{1+b \gamma_{s} \lambda_{i} f_{i}^{2}} .
$$

从信息传输的可靠性出发, 我们需要以 SER 上界的最小值为准则来进行功率分配, 于是 很容易建立下面约束条件下的最优化问题

$$
\varepsilon=\max _{f_{i}^{2}} \prod_{i=1}^{P}\left(1+b \gamma_{s} \lambda_{i} f_{i}^{2}\right) \quad \text { s.t. } \sum_{i=1}^{P} f_{i}^{2}=1 \text { and } f_{i}^{2}>0 .
$$

由于 $\ln (\cdot)$ 是一个单调递增函数, 为了便于求解 (27)式, 我们令 $\bar{\varepsilon}=\ln (\varepsilon)$ 且定义目标函数

$$
F=\sum_{i=1}^{P} \ln \left(1+b \gamma_{s} \lambda_{i} f_{i}^{2}\right)+\mu\left(\sum_{i=1}^{P} f_{i}^{2}-1\right),
$$

其中 $\mu$ 是 Lagrange 乘子. 将 $F$ 对 $f_{i}^{2}$ 求导, 并令其值等于 0 , 那么

$$
f_{i}^{2}=-\frac{1}{\mu}-\frac{1}{b \gamma_{s} \lambda_{i}}
$$

代入(27)式中的功率约束条件, 我们很容易得到

$$
-\frac{1}{\mu}=\frac{1}{P}\left(1+\frac{1}{b \gamma_{s}} \sum_{j=1}^{P} \frac{1}{\lambda_{j}}\right),
$$

于是第 $i$ 个波束的功率分配系数为

$$
f_{i}^{2}=\frac{1}{P}+\frac{1}{b \gamma_{s}}\left(\frac{1}{P} \sum_{j=1}^{P} \frac{1}{\lambda_{j}}-\frac{1}{\lambda_{i}}\right), \quad(i=1,2, \ldots, P) .
$$

在上式中矩阵 $\boldsymbol{R}$ 的特征值按非递增顺序排列确保了 $f_{1}^{2} \geqslant f_{2}^{2} \geqslant \cdots \geqslant f_{P}^{2}$. 然而, 对于给定 的功率 $E_{S}$, 功率分配系数必须满足 $f_{i}^{2}>0, \forall i \in[1, P]$, 因此由 $f_{P}^{2}>0$ 我们很容易求得形成 $P$ 个波束发射端所需要的最小 SNR 为

$$
\gamma_{s} \geqslant \frac{1}{b}\left(\frac{P}{\lambda_{P}}-\sum_{j=1}^{P} \frac{1}{\lambda_{j}}\right)^{\Delta}=\gamma_{\mathrm{th}, P} .
$$




\section{3 新的发射方案小结}

综上所述, 新的空时发射方案可以归纳为以下 5 步.

第 1 步: 每隔 $N_{b}$ 个数据块对信道估值 $\hat{\boldsymbol{h}}(i)$ 进行采样, 由得到的 $N_{T}$ 个信道响应矢量求得信 道自相关阵 $\boldsymbol{R}$ 的估计值

$$
\hat{\boldsymbol{R}}=\frac{1}{N_{T}} \sum_{n=1}^{N_{T}} \hat{\boldsymbol{h}}\left(n N_{b}\right) \hat{\boldsymbol{h}}^{\mathrm{H}}\left(n N_{b}\right),
$$

此处不失一般性, $N_{b}$ 的值足够大以保证 $\hat{\boldsymbol{h}}\left(n N_{b}\right)$ 和 $\hat{\boldsymbol{h}}\left((n+1) N_{b}\right)$ 互不相关.

第 2 步: 对 $\hat{\boldsymbol{R}}$ 进行特征值分解, 得到 $N$ 个非递增排列的特征值 $\lambda_{1} \geqslant \lambda_{2} \geqslant \cdots \geqslant \lambda_{N}$ 及对应的 特征矢量 $\boldsymbol{u}_{i}(i=1,2, \ldots, N)$.

第 3 步: 确定调制方式, 然后利用信道自相关阵 $\hat{\boldsymbol{R}}$ 的特征值和(34)式求得形成 $P$ 个波束的 信噪比门限值

$$
\gamma_{\mathrm{th}, P}=\frac{1}{b}\left(\frac{P}{\lambda_{P}}-\sum_{j=1}^{P} \frac{1}{\lambda_{j}}\right) \quad(P=1,2, \ldots, N) .
$$

第 4 步: 对于给定的功率 $E_{S}$, 如果 $\gamma_{S}=E_{S} / N_{0}$ 落在 $\left[\gamma_{\mathrm{th}, P}, \gamma_{\mathrm{th}, P+1}\right]$ 范围内, 那么就能确定 波束个数 $P$ 的值, 进一步利用(31)式求得功率分配系数 $f_{i}^{2}(i=1,2, \ldots, P)$.

第 5 步: 有了前面求得的 $\boldsymbol{u}_{i}$ 和 $f_{i}^{2}$, 由(15)式即可得到发射端自适应波束形成权矢量, 同时 结合 $P$ 的值确定正交空时块码的形式，从而完成了整个空时发射方案的设计.

\section{3 性能分析}

本节我们基于 MGF 和 Gauss-Chebyshev 积分对采用新方案的通信系统进行性能分析. 此 处跟前面一样, 我们仍然考虑 3 大类最为常用的调制方式.

\section{$3.1 M-\operatorname{PSK}(M \geqslant 4)$ 的错误概率}

将(12)和(21)式代入(17a)式, $M-P S K$ 调制下的 SER 可以近似表示为

$$
P_{S, \mathrm{PSK}} \approx 2 E\left[Q\left(\sqrt{2 \sin ^{2}\left(\frac{\pi}{M}\right) \gamma}\right)\right]=2 E\left[Q\left(\sqrt{2 \sin ^{2}\left(\frac{\pi}{M}\right) \lambda_{i} f_{i}^{2} \gamma_{s} \sum_{i=1}^{P}\left|\tilde{g}_{i}\right|^{2}}\right)\right] .
$$

令

$$
\zeta=2 \sin ^{2}\left(\frac{\pi}{M}\right) \gamma=2 \sin ^{2}\left(\frac{\pi}{M}\right) \lambda_{i} f_{i}^{2} \gamma_{s} \sum_{i=1}^{P}\left|\tilde{g}_{i}\right|^{2},
$$

那么 $M-P S K$ 的 SER 可通过下面的式子得到 ${ }^{[23]}$

$$
P_{S, \mathrm{PSK}} \approx 2 E[Q(\sqrt{\zeta})]=\frac{1}{j \pi} \int_{c-j \infty}^{c+j \infty} \Phi_{\zeta}(s)(2 s \sqrt{1-2 s})^{-1} \mathrm{~d} s,
$$

此处我们假设 $c$ 在 $\Phi_{\zeta}(s)(1-2 s)^{-1 / 2}$ 的收玫区域内, 通常取 $c=1 / 4 . \Phi_{\zeta}(s)$ 是 $\zeta$ 的 MGF, 它有如下 
的定义 23$]$

$$
\Phi_{\zeta}(s)=E[\exp (-s \zeta)] .
$$

将(36)式代入(38)式，并利用附录 $\mathrm{B}$ 的结果，我们可以得到

$$
\Phi_{\zeta}(s)=\prod_{i=1}^{P}\left[1+2 \sin ^{2}\left(\frac{\pi}{M}\right) s \lambda_{i} f_{i}^{2} \gamma_{s}\right]^{-1},
$$

更进一步令

$$
\Phi_{\Delta}(s)=\Phi_{\zeta}(s) \cdot(1-2 s)^{-1 / 2}=(1-2 s)^{-1 / 2} \prod_{i=1}^{P}\left[1+2 \sin ^{2}\left(\frac{\pi}{M}\right) s \lambda_{i} f_{i}^{2} \gamma_{s}\right]^{-1},
$$

以及 $s=c+j \omega$, 我们可以将(37)式表示成

$$
\begin{aligned}
P_{S, \mathrm{PSK}} & \approx 2 E[Q(\sqrt{\zeta})]=\frac{1}{j \pi} \int_{c-j \infty}^{c+j \infty} \frac{\Phi_{\Delta}(s)}{2 s} \mathrm{~d} s=\frac{1}{\pi} \int_{-\infty}^{+\infty} \frac{\Phi_{\Delta}(c+j \omega)}{c+j \omega} \mathrm{d} \omega \\
& =\frac{1}{\pi} \int_{-\infty}^{+\infty} \frac{c \operatorname{Re}\left[\Phi_{\Delta}(c+j \omega)\right]+\omega \operatorname{Im}\left[\Phi_{\Delta}(c+j \omega)\right]}{c^{2}+\omega^{2}} \mathrm{~d} \omega .
\end{aligned}
$$

其中 $\operatorname{Re}[\cdot]$ 和 $\operatorname{Im}[\cdot]$ 分别代表复数的实部和虚部. 进行变量替换 $\omega=c \sqrt{1-x^{2}} / x$ 后可以得到

$$
P_{S, \text { PSK }} \approx \frac{1}{2 \pi} \int_{-1}^{+1}\left\{\operatorname{Re}\left[\Phi_{\Delta}\left(c+j c \frac{\sqrt{1-x^{2}}}{x}\right)\right]+\frac{\sqrt{1-x^{2}}}{x} \operatorname{Im}\left[\Phi_{\Delta}\left(c+j c \frac{\sqrt{1-x^{2}}}{x}\right)\right]\right\} \frac{\mathrm{d} x}{\sqrt{1-x^{2}}},
$$

采用 $v$ 点的 Gauss-Chebyshev 数值积分后有

$$
P_{S, \mathrm{PSK}} \approx 2 E[Q(\sqrt{\zeta})]=\frac{1}{2 v} \sum_{k=1}^{v}\left\{\operatorname{Re}\left[\Phi_{\Delta}\left(c\left(1+j \tau_{k}\right)\right)\right]+\tau_{k} \operatorname{Im}\left[\Phi_{\Delta}\left(c\left(1+j \tau_{k}\right)\right)\right]\right\}+E_{v},
$$

其中 $\tau_{k}=\tan ((k-1 / 2) \pi / v)$, 并且当 $v \rightarrow \infty$ 时 $E_{v} \rightarrow 0$. 在数值计算中, 取 $v=64$, 其精度就能 满足要求. 更进一步, 假设采用的是 Gray 码, 那么误比特率(BER)可以表示为

$$
P_{B, \mathrm{PSK}} \approx \frac{1}{\log _{2} M} P_{S, \mathrm{PSK}} .
$$

\section{$3.2 M-P A M$ 的误符号率}

根据文献[21], 衰落信道下的 $M-P A M$ 误符号率可以表示为

$$
P_{S, \mathrm{PAM}}=\frac{2(M-1)}{M} E\left[Q\left(\sqrt{\frac{6}{M^{2}-1} \gamma}\right)\right]=\frac{2(M-1)}{M} E\left[Q\left(\sqrt{\frac{6}{M^{2}-1} \lambda_{i} f_{i}^{2} \gamma_{s} \sum_{i=1}^{P}\left|\tilde{g}_{i}\right|^{2}}\right)\right] .
$$

与前面 $M-P S K$ 的错误概率分析类似，令

$$
\zeta=\frac{6}{M^{2}-1} \lambda_{i} f_{i}^{2} \gamma_{s} \sum_{i=1}^{P}\left|\tilde{g}_{i}\right|^{2},
$$

那么 $\Phi_{\zeta}(s)$ 和 $\Phi_{\Delta}(s)$ 可以分别通过下面的式子得到 


$$
\Phi_{\zeta}(s)=\prod_{i=1}^{P}\left[1+\frac{6}{M^{2}-1} s \lambda_{i} f_{i}^{2} \gamma_{s}\right]^{-1}
$$

以及

$$
\Phi_{\Delta}(s)=(1-2 s)^{-1 / 2} \prod_{i=1}^{P}\left[1+\frac{6}{M^{2}-1} s \lambda_{i} f_{i}^{2} \gamma_{s}\right]^{-1} .
$$

利用 Gauss-Chebyshev 积分公式, 同时将(48)式代入(45)式, $M$-PAM 信号的 SER 就可以表示成

$$
\begin{aligned}
P_{S, \mathrm{PAM}} & =\frac{2(M-1)}{M} E\left[Q\left(\sqrt{\frac{6}{M^{2}-1} \gamma}\right)\right] \\
& =\frac{(M-1)}{2 M v} \sum_{k=1}^{v}\left\{\operatorname{Re}\left[\Phi_{\Delta}\left(c\left(1+j \tau_{k}\right)\right)\right]+\tau_{k} \operatorname{Im}\left[\Phi_{\Delta}\left(c\left(1+j \tau_{k}\right)\right)\right]\right\}+E_{v} .
\end{aligned}
$$

其中 $c=1 / 4, v=64, \tau_{k}=\tan ((k-1 / 2) \pi / v)$, 并且 $E_{v}=0$.

\section{3 正方形 $M-\mathrm{QAM}(M \geqslant 4)$ 的误符号率}

对于一个 $M$ 元, $M=2^{n}$ ( $n$ 为偶数)的正方形 QAM 调制信号, 由于可以将它看成是调制在 正交载波上的两路 $\sqrt{M}-\mathrm{PAM}$ 信号, 因此其误符号率为 ${ }^{[21]}$

$$
P_{S, \mathrm{QAM}}=1-\left(1-\bar{P}_{S, \mathrm{PAM}}\right)^{2},
$$

其中 $\bar{P}_{S, P A M}$ 表示 $\sqrt{M}$-PAM 信号的 SER. 直接利用前面 $M$-PAM 信号错误概率的分析结果, 我 们不难得到

$$
\begin{aligned}
\bar{P}_{\text {S,PAM }} & =2\left(1-\frac{1}{\sqrt{M}}\right) E\left[Q\left(\sqrt{\frac{3}{M-1} \gamma}\right)\right] \\
& =\frac{(\sqrt{M}-1)}{2 \sqrt{M} v} \sum_{k=1}^{v}\left\{\operatorname{Re}\left[\Phi_{\Delta}\left(c\left(1+j \tau_{k}\right)\right)\right]+\tau_{k} \operatorname{Im}\left[\Phi_{\Delta}\left(c\left(1+j \tau_{k}\right)\right)\right]\right\}+E_{v} .
\end{aligned}
$$

上式中的 $c=1 / 4, v=64, \tau_{k}=\tan ((k-1 / 2) \pi / v), E_{v}=0$, 以及

$$
\Phi_{\Delta}(s)=(1-2 s)^{-1 / 2} \prod_{i=1}^{P}\left[1+\frac{3}{M-1} s \lambda_{i} f_{i}^{2} \gamma_{s}\right]^{-1} \text {. }
$$

将(51)式代入(50)式, $M-\mathrm{QAM}(M \geqslant 4)$ 信号的误符号率就可直接计算得到.

\section{4 计算机仿真}

本节我们选择一个发射端采用均匀线阵, 且阵元间距离为半个波长, 而接收端采用单根 天线的通信系统进行计算机仿真. 在仿真过程中, 我们选择 $10^{\circ}$ 和 $60^{\circ}$ 两种典型的角度扩展值, 它们分别代表小角度扩展和大角度扩展 2 种情形, 在仿真结果图中用 Case 1 和 Case2 表示. 另 外, 我们只考虑 QPSK 和 16-QAM 两种调制方式, 而其他调制方式下的性能可由同样的方法得 到. 如果没有额外的说明, 信噪比定义成 $\mathrm{SNR}=E_{S} / N_{0}$. 
首先, 我们假设发射天线阵有 6 个单元, AOD 的均值与天线轴垂直, 即 $\theta_{c}=90^{\circ}$. 在 QPSK 调制下数值计算和仿真得到的 BER 性能随 $E_{b} / N_{0}$ (比特信噪比)的变化曲线如图 2 所示. 此处 的比特信噪比和(12)式中的符号信噪比之间有如下关系式

$$
\frac{E_{b}}{N_{0}}=\frac{1}{\log _{2} M} \frac{E_{S}}{N_{0}} .
$$

从图中我们可以看出, 在两种角度扩展情形下, 计算结果和仿真结果非常接近. 这意味着基于 MGF 和 Gauss-Chebyshev 积分的数值分析方法能获得满意的精度, 然而与 Monte-Carlo 仿真相 比较, 前者能大大地缩短仿真时间. 更进一步, 图中还给出了 BER 上界, 它与精确的 BER 形 状完全一致证明了我们以 SER 上界最小为设计准则的正确性.

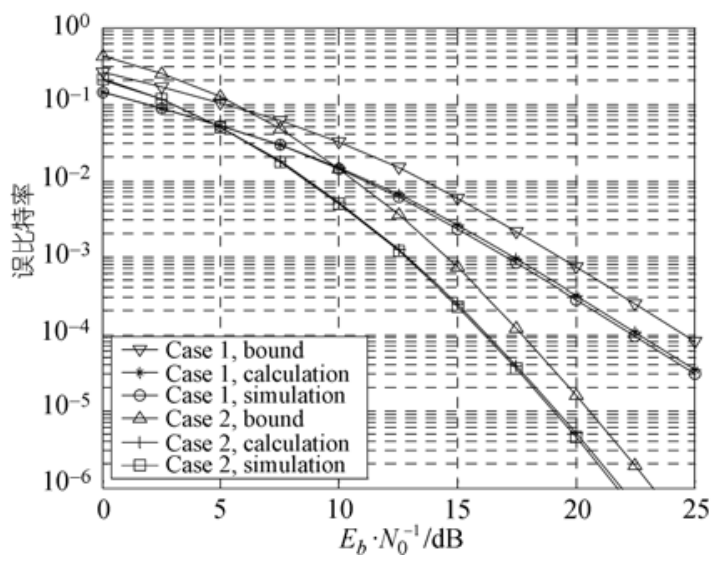

图 2 QPSK 调制下 BER 随 $E_{b} / N_{0}$ 的变化曲线

其次, 我们仍然采用前面的六元阵, 并且假设移动终端从 $120^{\circ}$ 扇区的一边移动到另一边, 从而覆盖整个扇区内的所有 AOD. 图 3 和 4 分别给出了采用 QPSK 和 16-QAM 两种调制方式 下, 平均误符号率(对整个扇区内的角度取平均)在 $10^{\circ}$ 和 $60^{\circ}$ 两种角度扩展下随 SNR 的变化曲 线. 不难发现, 在感兴趣的 SNR 区域内, 本文提出的方案在性能上优于其他的相关方法. 此处 其他方法是指经典的 Alamouti 空时块码 [7]、文献[14]提出的一维波束形成与空时块码相结合的 方法(用 $1 \mathrm{D} \mathrm{BF}+\mathrm{STBC}$ 表示)、文献[15]提出的二维波束形成与空时块码相结合的方法(用 $2 \mathrm{D}$ $\mathrm{BF}+\mathrm{STBC}$ 表示). 在小角度扩展情形下, 当 SNR 较低时, 本文方案所获得的性能与一维波束形 成方案几乎相同, 而随着 SNR 的增加, 它与二维波束形成方案非常接近. 可是在大角度扩展 情形下, 由于信道的自相关阵中有 2 个以上较大的特征值, 最佳功率分配和自适应波束形成使 得本文方案能获得更高的分集增益, 因而能明显改善其误符号性能. 更进一步, 从图中我们还 可以看出跟其他方案相比, 信噪比越高, 新方案的优势越明显.

最后，我们改变仿真条件如调制方式和角度扩展值，并重复上面的实验. 我们发现不管在 哪种情形下, 本文方案均能获得比其他相关方案优越的性能. 受篇幅所限, 在此我们不能给出 更多的仿真结果. 


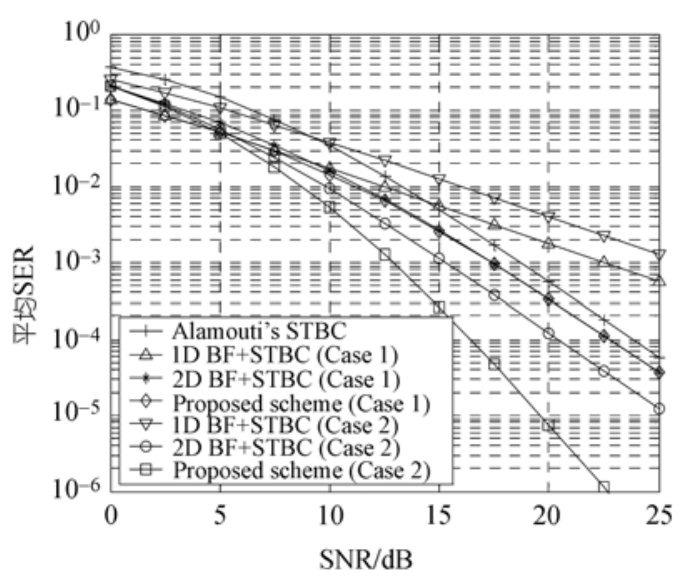

图 3 QPSK 调制下的平均 SER 性能曲线

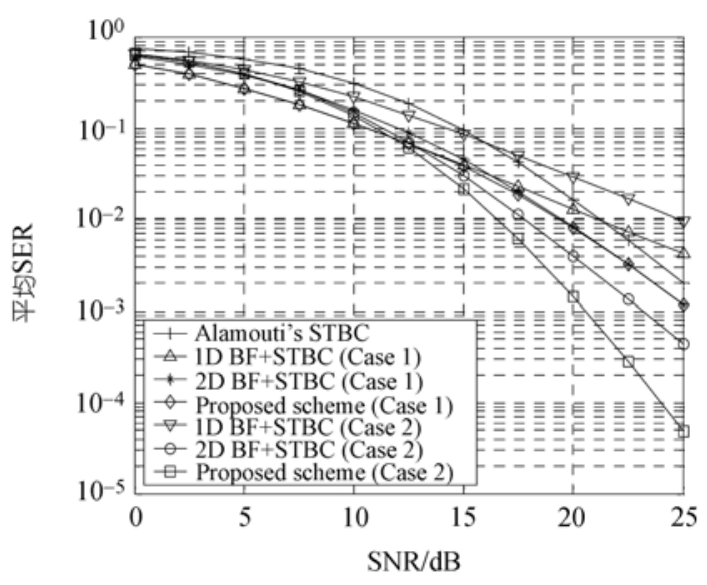

图 4 16-QAM 调制下的平均 SER 性能曲线

\section{5 结论}

近几年来, 波束形成与空时码, 尤其是空时块码, 相结合的方案在多天线通信中得到了广 泛的研究, 并被认为是提高无线通信系统性能的一种有效手段. 为了克服现有 BF 与 STBC 相 结合方案存在的缺点, 我们基于 STBC 所产生的等效加权 AWGN 信道模型, 并且以接收端达 到最大的平均 SNR 和最小的 SER 上界为设计准则, 提出了一种新的空时发射方案. 此外, 基 于 MGF 和 Gauss-Chebyshev 积分, 我们还提出了一种简单而精确的数值方法, 用来分析采用 新方案的通信系统在 3 大类调制方式下的性能. 最后计算机仿真结果证明了我们提出的下行 链路发射方案的性能优于常用的空时块码和现有相关文献上介绍的方法.

\section{参考文献}

1 Paulraj A, Gore D, Nabar R, et al. An overview of MIMO communications - a key to gigabit wireless. Proc IEEE, 2004, 92(2): 198-218 [DOI]

2 Foschini G J, Gans M J. On limits of wireless communications in a fading environment when using multiple antennas. Wirel Pers Commun, 1998, 6(3): 311-335 [DOI]

3 Telatar E. Capacity of multi-antenna Gaussian channels. Eur T Commun, 1999, 10(6): 585-595

4 Winters J H. Smart antennas for wireless systems. IEEE Pers Commun, 1998, 5(1): 23-27 [DOI]

5 Godara L C. Application of antenna arrays to mobile communications, part II: beam-forming and direction of arrival considerations. Proc IEEE, 1997, 85(8): 1195-1245 [DOI]

6 Tarokh V, Seshadri N, Calderbank A R. Space-time codes for high data rate wireless communication: performance criterion and code construction. IEEE T Inf Theor, 1998, 44(2): 744-765 [DOI]

7 Alamouti S M. A simple transmit diversity technique for wireless communications. IEEE J Sel Area Comm, 1998, 16(8): 1451-1458 [DOI]

8 Tarokh V, Seshadri N, Calderbank A R. Space-time block codes from orthogonal designs. IEEE T Inf Theor, 1999, 45(5): 1456-1467 [DOI]

9 Narula A, Lopez M J, Trott M D, et al. Efficient use of side information in multiple-antenna data transmission over fading channels. IEEE J Sel Area Comm, 1998, 16(8): 1423-1436 [DOI] 
10 Visotsky E, Madhow U. Space-time transmit precoding with imperfect feedback. IEEE T Inf Theor, 2001, 47(6): $2632-2639$ [DOI]

11 Jafar S A, Goldsmith A. Transmitter optimization and optimality of beamforming for multiple antenna systems. IEEE T Wirel Commun, 2004, 3(4): 1165-1175 [DOI]

12 Jöngren G, Skoglund M, Ottersten B. Combining beamforming and space-time block coding. IEEE T Inf Theor, 2002, 48(3): 611-627 [DOI]

$13 \mathrm{Vu} \mathrm{M}$, Paulraj A. Optimal linear precoders for MIMO wireless correlated channels with non-zero mean in space-time coded systems. IEEE T Signal Process, 2006, 54(6): 2318 - 2332 [DOI]

14 Liu J, Gunawan E. Combining ideal beamforming and Alamouti space-time block codes. Electron Lett, 2003, 39(17): $1258-1259 \underline{\text { [DOI] }}$

15 Lei Z, Chin F, Liang Y C. Combined beamforming with space-time block coding for wireless downlink transmission. Proc IEEE VTC, 2002: 2145-2148

16 Wang J, Ding P, Zoltowski M D, et al. Space-time coding and beamforming with partial channel state information. In: Proc IEEE Globecom. Saint Loui, 2005. 3149-3153

17 Zhou S, Giannakis G B. Optimal transmitter eigen-beamforming and space-time block coding based on channel correlations. IEEE T Inf Theor, 2003, 49(7): 1673-1690 [DOI]

18 Xu D, Huang Y, Yang L. Feedback of downlink channel state information based on superimposed coding. IEEE Commun Lett, 2007, 11(3): 240-242 [DOI]

19 Bengtsson M, Ottersten B. Optimal and suboptimal transmit beamforming. In: Godara L C, ed. Handbook of Antennas in Wireless Communications. New York: CRC Press LLC, 2001

20 Zhang H, Gulliver T A. Capacity and error probability analysis for orthogonal space-time block codes over fading channels. IEEE T Wirel Commun, 2005, 4(2): 808-819 [DOI]

21 Proakis J G. Digital Communications. 4th ed. New York: McGraw-Hill, 2001. 231-319

22 Vucetic B, Yuan J. Space-Time Coding. New York: John Wiley \& Sons Ltd, 2003. 49-90

23 Taricco G, Biglieri E. Exact pairwise error probability of space time codes. IEEE T Inf Theor, 2002, 48(2): 510$514 \underline{\text { DOI] }}$

24 Haykin S. Adaptive Filter Theory. 3rd ed. Englewood Cliffs, NJ: Prentice-Hall, 1996

\section{附录 A}

证明(13)式表示的约束条件下的最优化问题的解为(14)和(15)式.

将公式(9), (10)以及(13b)中的约束条件代入公式(13a), 目标函数就可以写成

$$
J=\max _{\boldsymbol{w}_{1}, \boldsymbol{w}_{2}, \ldots, \boldsymbol{w}_{P}} E\left[\|\boldsymbol{G}\|_{F}^{2}\right]=\max _{\boldsymbol{w}_{1}, \boldsymbol{w}_{2}, \ldots, \boldsymbol{w}_{P}} \sum_{i=1}^{P}\left(\boldsymbol{w}_{i}^{\mathrm{H}} \boldsymbol{R} \boldsymbol{w}_{i}\right)(P<N) .
$$

考虑到 $g_{i}=\boldsymbol{h}^{\mathrm{H}} \boldsymbol{w}_{i}$ 以及 $\boldsymbol{R}=E\left[\boldsymbol{h} \boldsymbol{h}^{\mathrm{H}}\right]$, 那么

$$
E\left[g_{i} \cdot g_{j}^{*}\right]=E\left[\boldsymbol{w}_{i}^{\mathrm{H}} \boldsymbol{h} \boldsymbol{h}^{\mathrm{H}} \boldsymbol{w}_{j}\right]=\boldsymbol{w}_{i}^{\mathrm{H}} \boldsymbol{R} \boldsymbol{w}_{j}=\boldsymbol{w}_{i}^{\mathrm{H}} \boldsymbol{U} \Sigma \boldsymbol{U}^{\mathrm{H}} \boldsymbol{w}_{j}=\sum_{n=1}^{N} \lambda_{n}\left(\boldsymbol{w}_{i}^{\mathrm{H}} \boldsymbol{u}_{n} \boldsymbol{u}_{n}^{\mathrm{H}} \boldsymbol{w}_{j}\right) \text {. }
$$

将约束条件 $E\left[g_{i} \cdot g_{j}^{*}\right]=0, \forall i \neq j$ 代入(A2)式, 不难得到

$$
\boldsymbol{w}_{i}^{\mathrm{H}} \boldsymbol{w}_{j}=0 \quad(\forall i \neq j) .
$$

为了采用 Lagrange 乘子法, 我们不妨定义代价函数

$$
F=\sum_{i=1}^{P}\left(\boldsymbol{w}_{i}^{\mathrm{H}} \boldsymbol{R} \boldsymbol{w}_{i}\right)+\sum_{i=1}^{P}\left(\frac{\mu_{i}}{f_{i}^{2}} \boldsymbol{w}_{i}^{\mathrm{H}} \boldsymbol{w}_{i}-1\right),
$$

其中 $\mu_{i}$ 表示 Lagrange 乘子. 将代价函数 $F$ 对 $\boldsymbol{w}_{i}$ 的共轭求梯度后, 不难得到 


$$
\frac{\partial F}{\partial \boldsymbol{w}_{i}^{*}}=\boldsymbol{R} \boldsymbol{w}_{i}+\frac{\mu_{i}}{f_{i}^{2}} \boldsymbol{w}_{i}
$$

上面的计算过程中用到了以下两个等式 $[24]$

$$
\frac{\partial\left(\boldsymbol{w}_{i}^{H} \boldsymbol{R} \boldsymbol{w}_{i}\right)}{\partial \boldsymbol{w}_{j}^{*}}= \begin{cases}\boldsymbol{R} \boldsymbol{w}_{i}, & i=j \\ 0, & i \neq j,\end{cases}
$$

和

$$
\frac{\partial\left(\boldsymbol{w}_{i}^{\mathrm{H}} \boldsymbol{w}_{i}\right)}{\partial \boldsymbol{w}_{j}^{*}}= \begin{cases}\boldsymbol{w}_{i}, & i=j, \\ 0, & i \neq j .\end{cases}
$$

令 $\partial F / \partial \boldsymbol{w}_{i}^{*}=\mathbf{0}$, 同时考虑到 $\boldsymbol{R}$ 的 $N$ 个特征值按非递增顺序排列, 且 $\boldsymbol{w}_{i} \neq \boldsymbol{w}_{j}(\forall i \neq j)$, 我们不难得到(A1) 式表示的约束条件下的最优化问题的解为

$$
\boldsymbol{w}_{i}^{\mathrm{opt}}=f_{i} \boldsymbol{u}_{i} .
$$

将(A8)式代入(A1)式，并经过简单的计算，则可进一步求得

$$
J=\sum_{i=1}^{P} \lambda_{i} f_{i}^{2} .
$$

\section{附录 B}

定理 1 假设 $\zeta=\alpha \sum_{i=1}^{N}\left\|\boldsymbol{H}_{i} \boldsymbol{Z}_{i}\right\|_{F}^{2}$, 其中 $\alpha$ 是一个复常数, $\boldsymbol{Z}_{i} \in C^{N \times 1}, \boldsymbol{H}_{i} \in C^{M \times N}$ 且它的每个元素都 是均值为 0 方差为 1 的复 Gauss 随机变量, 即 $\left[\boldsymbol{H}_{i}\right]_{j k} \sim \aleph_{C}(0,1)$. 定义 $\zeta$ 的 MGF 为

$$
\Phi_{\zeta}(s)=E[\exp (-s \zeta)]
$$

那么它的解析表达式为

$$
\Phi_{\zeta}(s)=\prod_{i=1}^{N}\left[1+\alpha s\left\|\boldsymbol{Z}_{i}\right\|_{F}^{2}\right]^{-M}
$$

证明 令

$$
\beta_{i}=\left\|\boldsymbol{H}_{i} \mathbf{Z}_{i}\right\|_{F}^{2},
$$

由于 $\left[\boldsymbol{H}_{i}\right]_{j k} \sim \aleph_{C}(0,1)$, 那么 $\beta_{i}$ 是一个以 $\left\|\boldsymbol{Z}_{i}\right\|_{F}^{2} / 2$ 为方差的 $\chi_{2 M}^{2}$ 分布, 其概率密度函数为 ${ }^{[21]}$

$$
p\left(\beta_{i}\right)=\frac{\beta_{i}^{M-1}}{\left(\left\|\boldsymbol{Z}_{i}\right\|_{F}^{2} / 2\right)^{M} 2^{M} \Gamma(M)} \exp \left(-\frac{\beta_{i}}{\left\|\boldsymbol{Z}_{i}\right\|_{F}^{2}}\right)=\frac{\beta_{i}^{M-1}}{\left(\left\|\mathbf{Z}_{i}\right\|_{F}^{2}\right)^{M} \Gamma(M)} \exp \left(-\frac{\beta_{i}}{\left\|\boldsymbol{Z}_{i}\right\|_{F}^{2}}\right),
$$

其中 $\Gamma(\cdot)$ 表示 Gamma 函数. 将(B3)式代入(B1)式, 那么 $\zeta$ 的 MGF 可由下面的式子得到

$$
\begin{aligned}
\Phi_{\zeta}(s) & =E\left[\exp \left(-\alpha s \sum_{i=1}^{N} \beta_{i}\right)\right]=\int_{0}^{\infty} \cdots \int_{0}^{\infty} \exp \left(-\alpha s \sum_{i=1}^{N} \beta_{i}\right) p\left(\beta_{1}\right) \cdots p\left(\beta_{N}\right) \mathrm{d} \beta_{1} \cdots \mathrm{d} \beta_{N} \\
& =\left[\int_{0}^{\infty} \exp \left(-\alpha s \beta_{1}\right) p\left(\beta_{1}\right) \mathrm{d} \beta_{1}\right] \cdots\left[\int_{0}^{\infty} \exp \left(-\alpha s \beta_{N}\right) p\left(\beta_{N}\right) \mathrm{d} \beta_{N}\right] .
\end{aligned}
$$

利用(B4)和(B5)式的第 $i$ 项可以写成

$$
\int_{0}^{\infty} \exp \left(-\alpha s \beta_{i}\right) p\left(\beta_{i}\right) \mathrm{d} \beta_{i}=\int_{0}^{\infty} \frac{\beta_{i}^{M-1}}{\left(\left\|\mathbf{Z}_{i}\right\|_{F}^{2}\right)^{M} \Gamma(M)} \exp \left[-\beta_{i}\left(\alpha s+\frac{1}{\left\|\boldsymbol{Z}_{i}\right\|_{F}^{2}}\right)\right] \mathrm{d} \beta_{i},
$$


令 $u=\beta_{i}\left(\alpha s+1 /\left\|\boldsymbol{Z}_{i}\right\|_{F}^{2}\right)$, 那么 $\beta_{i}=u /\left(\alpha s+1 /\left\|Z_{i}\right\|_{F}^{2}\right)$. 于是(B6)式又可以简化成

$$
\begin{aligned}
\int_{0}^{\infty} \exp \left(-\alpha s \beta_{i}\right) p\left(\beta_{i}\right) \mathrm{d} \beta_{i} & =\int_{0}^{\infty} \frac{u^{M-1} \exp (-u)}{\left(\alpha s+1 /\left\|\mathbf{Z}_{i}\right\|_{F}^{2}\right)^{M}\left(\left\|\mathbf{Z}_{i}\right\|_{F}^{2}\right)^{M} \Gamma(M)} \mathrm{d} u \\
& =\frac{1}{\left(1+\alpha s\left\|\mathbf{Z}_{i}\right\|_{F}^{2}\right)^{M} \Gamma(M)} \int_{0}^{\infty} u^{M-1} \exp (-u) \mathrm{d} u \\
& =\frac{1}{\left(1+\alpha s\left\|\mathbf{Z}_{i}\right\|_{F}^{2}\right)^{M}} .
\end{aligned}
$$

将(B7)式代入(B5)式，我们很容易得到 $\zeta$ 的 MGF 的表达式为

$$
\Phi_{\zeta}(s)=\prod_{i=1}^{N}\left[1+\alpha s\left\|\boldsymbol{Z}_{i}\right\|_{F}^{2}\right]^{-M} .
$$

